\title{
Antral follicle count quartile is an independent predictor of ongoing pregnancy in sub-optimal responders
}

\author{
Sezcan Mumusoglu MD'; Irem Yarali Ozbek MsC, MHC²; Mehtap Polat MD²; Gurkan \\ Bozdag MD1; Lale Karakoc Sokmensuer MD; Hakan Yarali MD ${ }^{1,2}$
}

\author{
${ }^{1}$ Hacettepe University School of Medicine, Department of Obstetric and Gynecology, Ankara, Turkey \\ ${ }^{2}$ Anatolia Women Health and IVF Centre, Ankara, Turkey \\ ${ }^{3}$ Hacettepe University School of Medicine, Department of Histology and Embryology, Ankara, Turkey
}

Objective: Sub-optimal response (SOR) is referred to as retrieval of 4-9 oocytes. However, 4-9 oocytes might be retrieved with from patients with different baseline ovarian reserve. Since there is paucity of data we aimed to assess the impact of baseline ovarian reserve on ICSI outcome in patients with SOR.

Methods: Retrospective cohort study. Inclusion criteria were: female age $<40$ yr-old, antral follicle count (AFC) $>7$ and chronologically the first cycle. Exclusion criteria were: azoospermia and pre-implantation genetic testing cycles. Ongoing pregnancy (OP; > 12 week of gestation) was taken as the primary outcome measure. A total of 568 consecutive couples undergoing ICSI cycle during Jan 20I4 - Jan 2017 were enrolled. GnRH-agonist and $\mathrm{GnRH}$-antagonist cycles were included; the starting dose of rec-FSH was 100-300 IU/day and adjusted according to the ovarian response. Quartiles of baseline antral follicle count (AFC) were formed; $Q<25^{\text {th }}$ $(<10), Q 25-75^{\text {th }}(10-16)$, and $\mathrm{Q}>75^{\text {th }}(>16)$. Logistic regression analysis was performed to delineate the independent significant predictors of OP.

Results: The $\mathrm{Q}>75^{\text {th }}$ arm was significantly younger and had higher body mass index (BMI) compared to the $Q<25^{\text {th }}$ and

Q25-75th arms (Table I).

\begin{tabular}{|c|c|c|c|c|}
\hline & $\begin{array}{l}Q<25^{\text {th }}(<10) \\
n=151\end{array}$ & $\begin{array}{l}Q^{25-75^{\text {th }}}(10-16) \\
n=291\end{array}$ & $\begin{array}{l}Q>75^{\text {th }}(>16) \\
n=126\end{array}$ & $P$ value \\
\hline Number of oocytes retrieved & $6.5 \pm 1.6^{\mathrm{a}}$ & $7.4 \pm 1.5^{\mathrm{c}}$ & $7.1 \pm 1.5^{b}$ & $<0.001$ \\
\hline Female age (yr) & $32.7 \pm 4.2^{\mathrm{c}}$ & $31.9 \pm 4.6^{\mathrm{c}}$ & $28.7 \pm 4.6^{\mathrm{a}}$ & $<0.001$ \\
\hline Body mass index $\left(\mathrm{kg} / \mathrm{m}^{2}\right)$ & $24.7 \pm 4.2^{\mathrm{c}}$ & $26.1 \pm 9.2$ & $27.4 \pm 5.6^{\mathrm{b}}$ & 0.009 \\
\hline Stimulation protocol, antagonist, $\mathrm{n}(\%)$ & $40(26.5)^{\mathrm{a}}$ & $114(39.2)^{\mathrm{a}}$ & $103(81.7)^{\mathrm{a}}$ & $<0.001$ \\
\hline Total gonadotropin dose (IU) & $2588.7 \pm 926.7^{\mathrm{a}}$ & $2207.2 \pm 876.1^{\mathrm{a}}$ & $1564.6 \pm 527.8^{\mathrm{a}}$ & $<0.001$ \\
\hline Number of embryo transferred & $1.5 \pm 0.5$ & $1.6 \pm 0.5$ & $1.5 \pm 0.5$ & 0.126 \\
\hline Rate of Day 5 transfer $\%$ & $46.5 \%$ & $45.6 \%$ & $45.0 \%$ & 0.802 \\
\hline Cycle cancelation rate, $\mathrm{n}(\%)$ & $8 / 151(5.3)$ & $21 / 291(7.2)$ & $7 / 126(5.5)$ & 0.676 \\
\hline Ongoing pregnancy rate per cycle, n (\%) & $43 / 151(28.5)^{\mathrm{c}}$ & $95 / 291(32.6)^{\mathrm{c}}$ & $59 / 126(46.8)^{\mathrm{a}}$ & 0.002 \\
\hline
\end{tabular}

a Statistically different from all other groups; ${ }^{b}$ Statistically different from $\mathrm{Q}<25^{\text {th }}$; c Statistically different from $\mathrm{Q}>75^{\text {th }}$
Although three groups fulfilled the definition of SOR, the mean number of oocytes was significantly less in the $Q<25^{\text {th }}$ arm. The total gonadotropin dose was significantly different in all three arms. Cycle cancellation rates and the mean number of embryos transferred were similar. However, there was a significant increase in OP per cycle with increasing AFC quartiles. When female age, BMI, indication of IVF, AFC quartile, ovarian stimulation protocol, number of retrieved oocytes, day 3/5 transfer, and number of transferred embryos were entered into the logistic regression model, only female age, AFC quartile, Day3/5 transfer and number of embryos transferred remained to be the significant predictors of OP. When $Q>75^{\text {th }}$ was taken as the reference, the OR $(95 \% \mathrm{Cl})$ for $\mathrm{Q}<25^{\text {th }}$ and $Q 25-75^{\text {th }}$ were $0.5(0.3-0.9 ; \mathrm{P}=0.029)$ and 0.6 $(0.4-0.9 ; p=0.040)$, respectively.

Conclusions: In sub-optimal responders, ovarian reserve, as assessed by AFC quartile, is an independent predictor of OP in fresh embryo transfer cycles. Increasing AFC is associated with significantly higher OP per started cycle in SOR patients, despite the transfer of comparable Day 3/5 transfer rate and number of embryos transferred. However, the impact of AFC quartile on cumulative pregnancy rates should be further studied. 